[BT2] -, - On the problem of $R$. Kadison and $I$. Singer, to appear.

[DMT] W. J. Davis, V. D. Milman and N. Tomczak-Jaegermann, The distance between certain n-dimensional Banach spaces, Israel J. Math. 39 (1981), 1-15.

[Fer] X Fernique, Régularité des trajectoires des fonctions aléatoires gaussiennes, in: Ecole d’été de probabilités de St. Flour IV-1974, Lecture Notes in Math. 480, Springer, 1975, 2-96.

[Gro] M. Gromov, Monotonicity of the volume of intersection of balls, in: Geometrical Aspects of Functional Analysis, Israel Seminar 1985-86, Lecture Notes in Math. 1267, Springer, 1987, 1-4.

[Kas] B. S. Ka shin, Some properties of matrices of bounded operators from $l_{2}^{[\mathrm{p}}$ into $l_{2}^{\mathrm{m}}, \mathrm{Izv}$. Akad. Nauk Armyan. SSR 15 (1980), 379-394 (in Russian)

[Kön] $\mathrm{H}$. Kö ni g, Type constants and $(q, 2)$-stumming norms defined by $n$ vectors, Isracl J. Math. 37 (1980), $130-138$

[KTJ] H. König and N. Tomczak-Jaegermann, Bounds for projection constants and 1-summing norms, Trans. Amer. Math. Soc. 320 (1990), 799-823.

[K-T] H. König and L. Tzafriri, Some estimates for type and cotype constants, Math. Ann. 256 (1981), 85-94.

[L-T] M. Ledoux and M. Ta lagrand, Comparison theorems, random geometry and some limit theorems for empirical processes, Ann. Probab. 17 (1989), 596-631.

[Lew] D. Lewis, Finite dimensional subspaces of $L_{p}$, Studia Math. 63 (1978), 207-212.

[Lun] A. A. Lunin, On operator norm of submatrices, Mat. Zametki 45 (3) (1989), 94-100 (in Russian).

[M-S] V. D. Milman and G. Schech tman, Asymptotic Theory of Finite Dimensional Normed Spaces, Lecture Notes in Math. 1200, Springer, 1986

[Peł] A. Pełczyński, Geometry of finite dimensional Banach spaces and operator ideals, in: Notes in Banach Spaces, E. Lacey (ed.), Univ. of Texas Press, 1981, 81-181.

[Pi1] G. Pisier, Remarques sur un résultat non publié de B. Maurey, Séminaire d'Analyse Fonctionnelle 1980-81, Exposé No. V, Ecole Polytechnique, Palaiseau.

[Pi2] -, On the duality between type and cotype, in: Martingale Theory in Harmonic Analysis and Banach Spaces, Proc. Cleveland 1981, Lecture Notes in Math. 939, Springer, 1982, 131-144.

[Pi3] -, Factorization of Linear Operators and Geometry of Banach Spaces, CBMS Regional Conf. Ser. in Math. 60, A.M.S., 1986.

[Tal] M. Talagrand, Embedding subspaces of $L_{1}$ into $I_{1}^{N}$, Proc. Amer. Math. Soc. 108 (1990), 363-369.

[TJ1] N. Tomczak-Ja egermann, Computing 2-summing norm with few vectors, Ark. Mat. 17 (1979), 273-277.

[TJ2] -, Banach-Mazur Distances and Finite-Dimensional Operator Ideals, Longman, 1989.

DEPARTMENT OF MATHEMATICS

CASE WESTERN RESERVE UNIVERSITY

Cleveland, Ohio 44106, USA.

\section{Every Radon-Nikodym Corson compact space is Eberlein compact}

\section{J. ORIHUELA (Murcia), W. SCHACHERMAYER (Linz) and M. VALDIVIA (Valencia)}

Abstract. We prove the result announced in the title. The Banach space version of this topological result reads as follows: A Banach space $E$ whose dual unit ball is a weak* Corson compact and which is GSG (i.e, there is an Asplund space $X$ and a continuous linear operator from $X$ into $E$ with dense range) is weakly compactly generated. We also analyze a relevant example of M. Talagrand and obtain solutions to three problems posed by I. Namioka.

1. Introduction. A compact topological space is called an Eberlein compact if it is homeomorphic to a weakly compact subset of some Banach space and is called Radon-Nikodym compact if it is homeomorphic to a weak* compact subset of the dual of an Asplund space. By the factorization result of [DFJP] every Eberlein compact space is homeomorphic to a weakly compact subset of a reflexive Banach space, therefore an Eberlein compact space is a RadonNikodym compact space. (For definitions and unexplained notation we refer to the end of the introduction.)

To see that these two notions are different, observe that for a compact scattered space $K$ the Banach space $C(K)$ is Asplund as the dual $C(K)^{*}$ equals $l^{1}(K)$ and therefore has RNP. Hence $K$ is a Radon-Nikodym compact space. For example the ordinal interval $\left[0, \omega_{1}\right]$ is a Radon-Nikodym compact but fails to be Eberlein (by Eberlein's theorem). Using the idea of dentability I. Namioka gave a topological characterization of Radon-Nikodym compacta [N] as those compact spaces which admit a lower semicontinuous fragmenting metric (see below). This gives rise to the notion of fragmented compacta ([JR], [N] where the lower semicontinuity assumption is dropped and we obtain the following chain of implications:

Eberlein compact $\Rightarrow$ Radon-Nikodym compact $\Rightarrow$ fragmented compact.

We shall prove in this paper that the second implication above also fails to be an equivalence.

1980 Mathemaics Subject Classification: Primary 46B22.

Key words and phrases: Eberlein compact, Radon-Nikodym compact, Corson compact, fragmentability, Asplund spaces. 
Another direction of generalizing the notion of Eberlein compacta was done in the work of $\mathrm{M}$. Talagrand [T] and S. Gul'ko [Gu3] which we summarize (as in [Ne]) by the following chain of implications, which all are known not to be equivalences (for definitions see below):

$$
\text { Eberlein } \Rightarrow \text { Talagrand } \Rightarrow \text { Gul'ko } \Rightarrow \text { Corson. }
$$

As regards the relationship between these two chains of implications it was shown in [G], [R] that

$$
\text { Gul'ko } \Rightarrow \text { fragmented space. }
$$

This implication summarizes the work done around the problem, posed by Talagrand, of whether every Gul'ko compact space has a dense $G_{\delta}$ metrizable subset. In [G] this question is positively answered and in [R] the spaces used by Gruenhage are seen to be fragmented compact spaces. Indeed, every fragmented compact space has a dense $G_{\delta}$ subset where the induced topology coincides with the topology induced by the fragmenting metric.

In [To], an example of a Corson compact space without dense metrizable subsets is given, so we know that

\section{Corson $\Rightarrow$ fragmented space.}

On the other hand, every scattered Corson compact space is an Eberlein compact space ([A], see also [V]).

The following questions related to the concepts above are posed in the paper [N] of Namioka:

PROBLEM 1. Find a compact Hausdorff space that is fragmented by a metric but is not Radon-Nikodym compact.

Problem 2. Do the implications Talagrand compact $\Rightarrow$ Radon-Nikodym compact or Gul'ko compact $\Rightarrow$ Radon-Nikodym compact hold true?

Problem 3. Find conditions for a Radon-Nikodym compact space to be Eberlein compact.

E. A. Reznichenko [K] has given an example of a Talagrand compact space which is not Radon-Nikodym compact, thus answering the questions posed in Problems 1 and 2. We show that Talagrand's initial example of a Talagrand compact space which is not Eberlein compact also fails to be Radon-Nikodym compact, thus also solving these two questions. We then proceed to prove the main result of this paper which is announced in the title, i.e. a Radon-Nikodym compact which is also Corson is already Eberlein. This gives in particular a complete solution to the first two questions and a rather general answer to Problem 3.

We shall use some embedding results which we summarize as follows: every compact Hausdorff space $K$ can be embedded into a cube $I^{T}$, where $I$ denotes the unit interval $[-1,+1]$, and $\Gamma$ is some set of continuous functions on $K$. $K$ is an Eberlein compact if and only if this embedding can be chosen to take values in $c_{0}(\Gamma)[\mathrm{AL}]$. By definition $K$ is a Corson compact if this embedding can be chosen to take values in the $\Sigma$-product space

$$
\Sigma(\Gamma)=\left\{x \in I^{\Gamma}:\{\gamma \in I: x(\gamma) \text { is not zero }\} \text { is countable }\right\} .
$$

$K$ is a Radon-Nikodym compact space if and only if this embedding can be done in such a way that the metric $d_{\Gamma}$ of uniform convergence on $\Gamma$ :

$$
d_{\Gamma}(x, y)=\sup \{|x(\gamma)-y(\gamma)|: \gamma \in \Gamma\} \text {, }
$$

$x$ and $y$ in $I^{r}$, fragments $K[\mathrm{~N}]$. Note that $d_{r}$ fragments $K$ if, and only if, for every countable subset $A$ of $\Gamma$ the pseudometric

$$
d_{A}(x, y)=\sup \{|x(\gamma)-y(\gamma)|: \gamma \in A\}
$$

is separable on $K$, which is parallel to Stegall's characterization of dual Banach spaces with the Radon-Nikodym property [S1].

Let us now give an outline of the organization of this paper:

In Section 2 we study in detail Talagrand's example of a Talagrand compact space $K$ which fails to be Eberlein. We show that it also fails to be Radon-Nikodym. Let us point out that the facts proved in Section 2 for the particular space $K$ will also follow from the general results obtained in the subsequent sections (which are logically independent of Section 2). Yet we recommend the reader to, first follow the arguments in the special case of Talagrand's example as many of the ideas used in the general case appear already here. Hence we make a point of giving detailed proofs for this special case; for example we explicitly define a fragmenting (not lower semicontinuous) metric on $K$.

In the subsequent sections we work on the proof of the main result of this paper, in its topological and Banach space versions:

THeorem A. A compact Hausdorff space is Eberlein if (and only if) it is Radon-Nikodym and Corson.

THeorem B. A Banach space $E$ is weakly compactly generated if (and only if) its dual unit ball is Corson compact and if it is GSG, i.e. there is a continuous linear map $T: X \rightarrow E$ with dense range, defined on an Asplund space $X$.

In Section 3 we reduce Theorem $B$ to the case where the Banach space $X$ is not only GSG but is already itself an Asplund space. This will imply that in the setting of Theorem A a compact space which is Radon-Nikodym and Corson can be weak* embedded into the dual of a Banach space $F$ which is Asplund and has weak* Corson compact dual unit ball. The technique of this section is to apply the factorization method of [DFJP] 
In Section 4 we show that a Banach space as above (i.e. one which is Asplund and has weak* Corson compact dual unit ball) is weakly compactly generated and therefore its dual unit ball is a weak* Eberlein compact. This will be proved with the help of "long sequences of projections" as developed in [V].

Finally, in Section 5 we pull the strings together and give the proofs for Theorems $\mathbf{A}$ and $\mathbf{B}$.

Similar. results have been obtained at about the same time by C. Stegall. We now collect the notions used in this paper:

If $(X, \mathscr{T})$ is a topological space and $d$ is a metric on $X$, which need not be related to $\mathscr{T}$ in any way, we say that the space $(X, \mathscr{T})$ is fragmented by $d$ if, for each nonempty subset $A$ of $X$ and for each positive $\varepsilon$, there exists a $\mathscr{T}$-open subset $U$ of $X$ such that $U \cap A$ is nonvoid and $d$-diam $(U \cap A) \leqslant \varepsilon$.

A compact Hausdorff space $(X, \mathscr{T})$ is called fragmented (resp. RadonNikodym) if there is a metric (resp. a $\mathscr{T}$-1.s.c. metric) $d$ on $X$ fragmenting $(X, \mathscr{T})$.

A topological space $(X, \mathscr{T})$ is countably determined (resp. $K$-analytic) if there is a metrizable and separable (resp. complete, metrizable and separable) space $P$ and a set-valued map $T$ from $P$ onto $X$ with compact values and upper semicontinuous.

A compact topological space is called a Gul'ko (resp. Talagrand) compact if it is homeomorphic to a weak* compact subset of the dual of a weakly countably determined (resp. weakly $K$-analytic) Banach space.

If $(X, \mathscr{T})$ is a topological space, we denote by $C_{\mathrm{p}}(X)$ the space of all real continuous functions defined on $X$ endowed with the pointwise topology. A compact topological space $K$ is Gul'ko (resp. Talagrand) if, and only if, $C_{\mathrm{p}}(K)$ is countably determined (resp. $K$-analytic).

We denote by $\omega_{0}$ the first infinite ordinal. For a topological space $(X, \mathscr{T})$ the density character, $\operatorname{dens}(X)$, is the first cardinal $\lambda$ such that there is a dense subset $A$ of $(X, \mathscr{T})$ with cardinality $\lambda$. For a set $A$, we denote by $|A|$ its cardinal number.

A projectional resolution of identity on a Banach space $E$ is a "long sequence of projections" $\left\{P_{\alpha}: \omega_{0} \leqslant \alpha \leqslant \mu\right\}$, where $\mu$ is the first ordinal whose cardinality equals the density character of $E$, which satisfies:

(i) $\left\|P_{\alpha}\right\|=1$ for every $\alpha$.

(ii) $P_{\alpha} P_{\beta}=P_{\alpha}=P_{\beta} P_{\alpha}$ if $\omega_{0} \leqslant \alpha \leqslant \beta \leqslant \mu$.

(iii) $\operatorname{dens}(P(E)) \leqslant|\alpha|$ for every $\alpha$.

(iv) $\bigcup\left\{P_{\beta+1}(E): \beta<\alpha\right\} \|=P_{\alpha}(E)$ for every $\alpha$

(v) $P_{\mu}$ is the identity operator on $E$.

A projectional resolution of identity $\left\{P_{\alpha}: \omega_{0} \leqslant \alpha \leqslant \mu\right\}$ is called a shrinking resolution when the adjoint projections $\left\{P_{\alpha}^{*}: \omega_{0} \leqslant \alpha \leqslant \mu\right\}$ form a resolution of identity for the dual space $E^{*}$.

2. Talagrand's example is not a Radon-Nikodym compact. Let $K$ be a subset of the cube $I^{T}$. We say that $K$ is solid if it satisfies the following condition: If $x \in K, y \in I^{r}$ and

then $y \in K$.

$$
y(\gamma)=x(\gamma) \text { or } y(\gamma)=0 \text { for every } \gamma \text { in } \Gamma,
$$

Propostrion 2.1. Let $K$ be a solid compact subset of $I^{\Gamma}$. The metric

$$
d_{\Gamma}(x, y)=\sup \{|x(\gamma)-y(\gamma)|: \gamma \in \Gamma\}, \quad x \text { and } y \text { in } I^{\Gamma},
$$

fragments $K$ if, and only if, $K$ is contained in $c_{0}(\Gamma)$.

Proof. If $K$ is fragmented by $d_{r}$, then every $x$ in $K$ belongs to $c_{0}(\Gamma)$. Otherwise, we could find a countable infinite subset $A$ of $\Gamma$, an $\varepsilon>0$ and a point $x$ in $K$ such that $|x(\gamma)|>\varepsilon$ for every $\gamma$ in $A$. For every subset $P$ of $A$ we define a point $x_{p}$ of $K$ by $x_{P}(\gamma)=x(\gamma)$ if $\gamma \in P$ and $x_{P}(\gamma)=0$ otherwise. The subset of $K$ defined by $\left\{x_{P}: P \subset A\right\}$ is uncountable and such that $d_{A}\left(x_{P}, x_{Q}\right)$ $>\varepsilon$ if $P \neq Q$. Therefore, $d_{A}$ is not separable on $K$ and the metric $d_{\Gamma}$ does not fragment $K$ because of Namioka's theorem [N] mentioned in the introduction.

On the other hand, every weakly compact subset of a Banach space is fragmented by the norm; if $K$ is contained in $c_{0}(\Gamma)$ then $K$ is a weakly compact subset of it and the norm of $c_{0}(\Gamma)$ induces the metric $d_{\Gamma}$ on $K$.

There is a class of solid compact spaces which has been used for the construction of suitable examples. This is the class of spaces that result from an adequate family of sets.

Definition ([T], [P]). A family $\mathbb{C}$ of subsets of a nonempty set $T$ is said to be adequate if:

(i) $A \in \mathbb{C}$ and $B \subseteq A$ implies $B \in \mathbb{C}$;

(ii) if $A$ is a subset of $T$ such that every finite subset of $A$ belongs to $\mathbb{C}$ then $A$ belongs to $\mathbb{C}$;

(iii) for every point $t$ in $T$ the set $\{t\}$ belongs to $\mathcal{C}$

Given an adequate family $\mathfrak{C}$, the set of characteristic functions

$$
K(\mathbb{C})=\left\{\chi_{A^{*}}: A \in \mathbb{C}\right\}
$$

is a compact subset of the product space $\{0,1\}^{T}$. We often identify $A$ with $\chi_{A}$, and thus consider $K$ as a space of subsets of $T$ equipped with the topology $\tau$ inherited from $\{0,1\}^{T}$.

TALAGRAND's EXAMPLE. We shall work with the set $T=\mathbf{N}^{\mathrm{N}}$ of sequences of positive integers together with the adequate families of subsets given by

$$
\mathscr{U}_{0}=\left\{\{\sigma\}: \sigma \in \mathbf{N}^{\mathbf{N}}\right\} \cup \varnothing,
$$


and for every positive integer $n$

$$
\mathfrak{U}_{n}=\left\{A \subset \mathbf{N}^{\mathbf{N}}: \sigma, \varrho \in A \text { and } \sigma \neq\left.\varrho \Rightarrow \sigma\right|_{n}=\left.\varrho\right|_{n} \text { and }\left.\sigma\right|_{n+1} \neq \varrho l_{n+1}\right\}
$$

where for each sequence $\sigma$ in $\mathbf{N}^{\mathbf{N}}$ and each positive integer $n$,

$$
\left.\sigma\right|_{n}=(\sigma(1), \ldots, \sigma(n)) \text {. }
$$

We denote by $K_{0}$ the compact set $K\left(\mathfrak{A}_{0}\right)$ and by $K_{n}$ the compact set $K\left(\mathfrak{U}_{n}\right)$ for every positive integer $n$. We also define $\mathfrak{Q}=\bigcup\left\{\mathfrak{X}_{n}: n=1,2, \ldots\right\}$ which is also an adequate family and gives us Talagrand's example $K$ :

$$
K=K(\mathfrak{A})=\bigcup\left\{K_{n}: n=1,2, \ldots\right\} .
$$

Observe that $\mathfrak{U}_{n} \cap \mathfrak{U}_{m}=\mathfrak{U}_{0}$ if $n \neq m$, and so $K_{n} \cap K_{m}=K_{0}$ if $n \neq m$.

THEOREM 2.2. The compact space $K$ is a Talagrand compact that admits a fragmenting metric and is not a Radon-Nikodym compact space.

We begin with some intuitive arguments which suggest that it is not possible to find any lower semicontinuous metric fragmenting $K$.

Identifying $(K, \tau)$ with a subset of $\left(l^{\infty}\left(\mathbf{N}^{\mathbf{N}}\right), \sigma^{*}\right)$ consider the metric $\varrho_{0}$ on $K$ induced by the norm \|\|$_{\infty}$ of $l^{\infty}\left(\mathbf{N}^{N}\right)$ which is nothing but the discrete metric on $K$.

Note that $\left(K_{0}, \tau\right)$ is homeomorphic to the one-point compactification of the discrete set $\mathbf{N}^{\mathbf{N}}$ (the point at infinity is the point 0 ) and therefore the discrete metric $\varrho_{0}$ on $K_{0}$ fragments $K_{0}$. (This is easily seen directly; it may also be deduced more abstractly from the fact that $K_{0} \subset c_{0}\left(\mathbf{N}^{N}\right)$ and from the fragmentability of weakly compact sets.)

But $\varrho_{0}$ does not fragment $K$ and in fact it does not fragment any $K_{n}$, for $n \geqslant 1$, as follows from the above proposition.

In our search for a $\tau$-1.s.c. metric on $K$ which fragments all of $K$ (this search will turn out to be unsuccessful) a first step would be to find, for $n \in \mathbf{N}$, a $\tau$-1.s.c. metric $\varrho_{n}$ on $K$ which fragments $K_{0} \cup \ldots \cup K_{n}$. This can indeed be done: Define

$$
\begin{gathered}
T_{n}: l^{\infty}\left(\mathbf{N}^{N}\right) \rightarrow l^{\infty}\left(\mathbf{N}^{N}\right), \\
T_{n} f(\sigma)=((\sigma(1)+1) \ldots(\sigma(n+1)+1))^{-1} f(\sigma), \quad f \in l^{\infty}\left(\mathbf{N}^{N}\right), \sigma \in \mathbf{N}^{N} .
\end{gathered}
$$

Then $T_{n}$ is a weak* continuous injective map from $l^{\infty}\left(\mathrm{N}^{\mathrm{N}}\right)$ into itself such that $T_{n}\left(K_{0} \cup \ldots \cup K_{n}\right) \subseteq c_{0}\left(\mathbf{N}^{N}\right)$. Hence the metric

$$
\varrho_{n}(A, B)=\left\|T_{n}\left(\chi_{A}\right)-T_{n}\left(\chi_{B}\right)\right\|_{\infty}, \quad A, B \in K,
$$

is a l.s.c. metric on $(K, \tau)$ that fragments $K_{0} \cup \ldots \cup K_{n}$.

But the crux is that $\varrho_{n}$ does not fragment $K_{n+l}$ for $l \in \mathrm{N}$ as one deduces from the above proposition. On the other hand, the metric $\varrho_{n+1}$ does fragment $K_{n+1}$ too, but the reader should note that $\varrho_{n+1}$ induces a strictly coarser metric than $\varrho_{n}$ on $K_{n}$.
The obvious thing to do now is to paste the $\varrho_{n}$ 's together in such a way as to obtain a metric $\varrho$ which fragments all of $K$. For example, one may define

$$
\varrho(A, B)= \begin{cases}\varrho_{n}(A, B) & \text { if } A \text { and } B \text { are in } K_{n} \backslash K_{0}, \\ 1 & \text { otherwise. }\end{cases}
$$

This metric indeed fragments all of $K$ : Let $F \subseteq K$ be nonvoid. Either $F \subseteq K_{0}$, in which case $F$ contains a $\tau$-open singleton; or there is a positive integer $n$ such that $F \cap\left(K_{n} \backslash K_{0}\right) \neq \varnothing$. In the latter case we can find, for $\varepsilon>0$, a $\tau$-open subset $V$ of $K$ contained in $K_{n} \backslash K_{0}$ such that $V \cap F \neq \varnothing$ and $Q-\operatorname{diam}(V \cap F)=\varrho_{n}$-diam $(V \cap F)<\varepsilon$

However, the problem with the above metric $\varrho$ is that - unlike the metrics $\Omega_{n}$-it fails to be $\tau-$-l.s.c. Let us see why: Fix two distinct points $\mu$ and $\sigma$ in $K_{0}$, both different from 0 , and let $\left(A_{n}\right)_{n=1}^{\infty}$ and $\left(B_{n}\right)_{n=1}^{\infty}$ be elements of $K_{n} \backslash K_{0}$ such that $A_{n}$ contains $\mu$, so it starts at $\left.\mu\right|_{n}$, and $B_{n}$ contains $\sigma_{3}$, so it starts at $\left.\sigma\right|_{n}$; i.e. for $\alpha \in A_{n},\left.\alpha\right|_{n}=\left.\mu\right|_{n}$ and for $\beta \in B_{n},\left.\beta\right|_{n}=\left.\sigma\right|_{n}$. Then

$$
\tau-\lim _{n} A_{n}=\{\mu\}, \quad \tau-\lim _{n} B_{n}=\{\sigma\}
$$

and

$$
\lim _{n} \varrho\left(A_{n}, B_{n}\right)=\lim _{n} \varrho_{n}\left(A_{n}, B_{n}\right)=0,
$$

which readily shows that $\varrho$ fails to be $\tau$-l.s.c. in view of $\varrho(\{\mu\},\{\sigma\})=1$.

Summing up what we have shown so far about Talagrand's space $K$ : There is a fragmenting metric on $K$, namely $\varrho$, but this metric fails to be $\tau$-l.s.c. In the rest of this section we shall show that no fragmenting metric $d$ on $K$ can be $\tau$-1.s.c. for reasons which are essentially the same as the argument given for $Q$ above. This will prove Theorem 2.2 .

So suppose that there is a $\tau$-l.s.c. fragmenting metric $d$ on $K$ and let us work towards a contradiction:

The metric $d$ induces a topology on $K$ finer than $\tau$ [JNR]. Thus the identity from $\left(K_{0} \backslash\{0\}, d\right)$ into $\left(K_{0} \backslash\{0\}, \tau\right)$ is continuous and the last space has the discrete topology. So this is an homeomorphism, whence for every $\sigma$ in $\mathbf{N}^{\mathbf{N}}$ there is a positive number $\delta_{\sigma}$ such that

$$
d(\{\sigma\}, x)>\delta_{\sigma}>0
$$

for every $x$ in $K_{0}$. In what follows we shall work with the numbers $\delta_{\sigma}$ and we shall see how, in some sense, the discreteness of $K_{0}$ spreads over all of $K$. We need some preparatory lemmas:

LEMMA 1. For every $\sigma$ in $\mathrm{N}^{\mathrm{N}}$ there is a positive integer $n_{\sigma}$ such that if $A$ and $B$ are elements of $K_{m}, m \geqslant n_{\sigma}$ and $\sigma \in(A \backslash B) \cup(B \backslash A)$, then $d(A, B) \geqslant \delta_{\sigma} / 2$.

Proof. It is enough to note that any sequence $\left(A_{n}\right)$ in $K$ with $A_{n}$ in $K_{n}$ has a cluster point in $K_{0}$. Then arguing by contradiction we get the conclusion from the lower semicontinuity of the metric $d$ and the definition of the numbers $\delta_{a}$. 
Indeed, suppose that the assertion does not hold true. We can find an increasing sequence of positive integers $m_{1}<m_{2}<\ldots$ and subsets $A_{n}$ and $B_{n}$ in $K_{m_{n}}$ such that $\sigma \in A_{n}, \sigma \notin B_{n}$, and

$$
d\left(A_{n}, B_{n}\right)<\delta_{\sigma} / 2 .
$$

The sequence $\left(A_{n}\right)$ converges to $\{\sigma\}$ in $K$ and $\left(B_{n}\right)$ has a cluster point $x$ in $K_{0}$ different from $\{\sigma\}$. By the lower semicontinuity of $d$, we know that $d(\{\sigma\}, x) \leqslant \delta_{\sigma} / 2$, which contradicts the previous choice of the number $\delta_{\sigma}$.

We shall now work with the product space $\mathbf{N}^{\mathbf{N}}$ of discrete spaces $\mathbf{N}$. Note that if a subset $\mathfrak{H}$ of $\mathbf{N}^{\mathbf{N}}$ is not contained in a $\sigma$-compact subset and $\mathfrak{H}=\bigcup\left\{\mathfrak{H}_{n}: n=1,2, \ldots\right\}$, then there is a positive integer $r$ such that $\mathfrak{S}_{r}$ is not contained in a $\sigma$-compact subset.

LEMma 2. There exists a subset $\Sigma^{\prime} \subseteq \mathbf{N}^{\mathbf{N}}$, which is not contained in a $\sigma$-compact subset, and positive integers $p$ and $q$, such that for any $A$ and $B$ in $K_{m}$ with $m \geqslant q$ and $[(A \backslash B) \cup(B \backslash A)] \cap \Sigma^{\prime}$ nonvoid

$$
d(A, B)>1 /(2 p) \text {. }
$$

Proof. Consider the identity

$$
\mathbf{N}^{\mathbf{N}}=\bigcup\left\{\left\{\sigma \in \mathbf{N}^{\mathbf{N}}: \delta_{\sigma}>1 / n \text { and } n_{\sigma}<k\right\}: n, k=1,2, \ldots\right\},
$$

where $n_{\sigma}$ is given by Lemma 1 above. As $\mathbf{N}^{\mathbf{N}}$ is not $\sigma$-compact, there are positive integers $p$ and $q$ such that

$$
\Sigma^{\prime}=\left\{\sigma \in \mathbf{N}^{\mathbf{N}}: \delta_{\sigma}>1 / p \text { and } n_{\sigma}<q\right\}
$$

is not contained in a $\sigma$-compact subset.

LEMmA 3. Let $\mathfrak{T}$ be a subset of $\mathbf{N}^{\mathbf{N}}$ which is not contained in a $\sigma$-compact subset and $q$ a fixed positive integer. Then there is a finite sequence $\left(s_{1}, \ldots, s_{k}\right)$ with $k \geqslant q$ such that

is infinite.

$$
\left\{n \in \mathbf{N} \text { : for some } \sigma \text { in } \mathfrak{I},\left.\sigma\right|_{k+1}=\left(s_{1}, \ldots, s_{k}, n\right)\right\}
$$

Proof. Fix $q$ and write

$$
\mathfrak{I}=\bigcup\left\{\left\{\sigma \in \mathfrak{T}:\left.\sigma\right|_{q}=\left(b_{1}, \ldots, b_{q}\right)\right\}:\left(b_{1}, \ldots, b_{q}\right) \in \mathbf{N}^{q}\right\} .
$$

Since $\mathfrak{I}$ is not contained in a $\sigma \sim$ compact subset, there is some $\left(a_{1}, \ldots, a_{t}\right) \in \mathrm{N}^{4}$ such that

$$
\mathfrak{I}_{q}:=\left\{\sigma \in \mathfrak{I} ;\left.\sigma\right|_{q}=\left(a_{1}, \ldots, a_{q}\right)\right\}
$$

is not contained in a $\sigma$-compact subset. Now suppose that the assertion of the lemma is false. Then the set

$$
\begin{aligned}
& \left\{m \in \mathbf{N}: \text { for some } \sigma \in \mathfrak{I}_{q}, \sigma(q+1)=m\right\} \\
& =\left\{m \in \mathbf{N}: \text { for some } \sigma \in \mathfrak{T},\left.\sigma\right|_{q+1}=\left(a_{1}, \ldots, a_{q}, m\right)\right\}
\end{aligned}
$$

is finite. Let $a_{q+1}$ denote the maximum of this set. So we have

$$
\mathfrak{I}_{q} \subset\left\{\sigma \in \mathfrak{T}: \sigma(i) \leqslant a_{i}, i=1, \ldots, q+1\right\} .
$$

Proceeding by recurrence, suppose we have found integers $a_{q+1}, \ldots, a_{q+n}$ such that

$$
\mathfrak{T}_{q} \subset\left\{\sigma \in \mathfrak{I}: \sigma(i) \leqslant a_{i}, i=1, \ldots, q+n\right\} .
$$

For every finite sequence $\left(s_{1}, \ldots, s_{q+n}\right)$ with $s_{i} \leqslant a_{i}, i=1, \ldots, q+n$, the set

$$
\left\{m \in \mathbf{N} \text { : for some } \sigma \in \mathfrak{T},\left.\sigma\right|_{q+n+1}=\left(s_{1}, \ldots, s_{q+n}, m\right)\right\}
$$

is finite, and we denote it by $N\left(s_{1}, \ldots, s_{q+n}\right)$. The set of integers

$$
\bigcup\left\{N\left(s_{1}, \ldots, s_{q+n}\right): s_{i} \leqslant a_{i}, i=1, \ldots, q+n\right\}
$$

is finite and we can find its maximum, which we denote by $a_{q+n+1}$. We thus have

$$
\mathfrak{I}_{q} \subset\left\{\sigma \in \mathfrak{T}: \sigma(i) \leqslant a_{i}, i=1, \ldots, q+n+1\right\} .
$$

Finally, we get a sequence $\left(a_{n}\right)$ in $\mathbf{N}^{\mathbf{N}}$ such that $\mathfrak{I}_{q}$ is contained in the compact set $\left\{\sigma \in \mathfrak{T}: \sigma(i) \leqslant a_{i}, i=1,2, \ldots\right\}$, which is a contradiction.

Proof of Theorem 2.2. We apply Lemma 3 to the set $\Sigma^{\prime}$ and the positive integer $q$ given by Lemma 2 . There exists a finite sequence $\left(s_{1}, \ldots, s_{k}\right)$ with $k \geqslant q$ such that

$$
\left\{n \in \mathbf{N}: \text { for some } \sigma \in \Sigma^{\prime},\left.\sigma\right|_{k+1}=\left(s_{1}, \ldots, s_{k}, n\right)\right\}
$$

is an infinite subset of $\mathbf{N}$, which we denote by $P$.

Denote by $F$ the Banach space of all continuous and $d$-Lipschitz functions on $K_{k}$ with the norm \|\|$_{\text {Lip }}+\|\|_{\infty}$. Then $K_{k}$ is a weak* compact subset of the dual $F^{*}$, and the dual norm also fragments $K_{k}$ according to a theorem of Ghoussoub and Maurey [GM, Th. VII.1] (see also [JNR]). Now, if $\sigma \in \mathbf{N}^{\mathbf{N}}$ and $e_{\sigma}$ denotes the canonical projection from $\{0,1\}^{\mathrm{N}}$ onto the coordinate $\sigma$, then the family of functions

$$
\mathfrak{F}=\left\{(1 /(2 p)) e_{a}: \sigma \in \Sigma^{\prime}\right\},
$$

where $p$ is given by Lemma 2 , is a bounded subset of $F$. Indeed, the only thing to check is the Lipschitz condition. Take a pair of elements $A$ and $B$ in $K_{k}$ and $\sigma$ a fixed element in $\Sigma^{\prime}$. Then we have

$$
\begin{array}{ll}
\left|e_{\sigma}(A)-e_{\sigma}(B)\right|=0 & \text { if } \sigma \in A \cap B \text { or } \sigma \notin A \cup B, \\
\left|e_{\sigma}(A)-e_{\sigma}(B)\right|=1 & \text { if } \sigma \in(A \backslash B) \cup(B \backslash A) .
\end{array}
$$

In each case, from Lemma 2 , we have

$$
\left|(1 /(2 p)) e_{\sigma}(A)-(1 /(2 p)) e_{\sigma}(B)\right| \leqslant d(A, B)
$$

because $d(A, B)>1 /(2 p)$ if $\sigma \in(A \backslash B) \cup(B \backslash A)$. Now we arrive at a contradiction: 
Let $R$ be the restriction mapping from $[0,1]^{N^{N}}$ into $[0,1]^{\mathfrak{T}}$. We obtain a solid compact.subset $R\left(K_{k}\right)$ of the cube $[0,1]^{\mathfrak{F}}$ which is fragmented by $d_{\mathfrak{F}}$, but which is not contained in $c_{0}(\tilde{F})$. Indeed, for every $q$ in $P$ there is some $\sigma_{q}$ in $\Sigma^{\prime}$ with the first $k+1$ coordinates equal to $\left(s_{1}, \ldots, s_{k}, q\right)$, and the set $A=\left\{\sigma_{q}: q \in P\right\}$ belongs to $K_{k}$ and $R(A)$ is not in $c_{0}(\mathfrak{F})$.

Remark. We shall see that the arguments given for Talagrand's example above are already essentially applicable to the general case, i.e. the impossibility of a Corson compact to be Radon-Nikodym without being already Eberlein.

Let us. indicate briefly why: By its very definition Talagrand's space is a subset of $\Sigma\left(\mathbf{N}^{N}\right)$, whence it is obvious that it is a Corson compact. What we have done in the above proof-assuming $K$ is a Radon-Nikodym compact - is the following: We have embedded $K$ (or at least $K_{k}$ ) into the dual of a Banach space $F$ such that $K$ is fragmented by the norm of $F^{*}$ and such that the coordinate functionals $e_{o}$ on $K$ are in $F$.

In the general case of a Corson compact $K$ we shall proceed similarly: Represent $K$ as a subset of $\Sigma(\Gamma)$ for some set $\Gamma$ and-assuming $\bar{K}$ is a Radon-Nikodym compact space-embed it into a dual Banach space $F^{*}$ with the Radon-Nikodym property. But we have to do this in such a way that the coordinate functionals $\left(e_{\gamma}: \gamma \in \Gamma\right)$ are contained in $F$. To do so we apply the interpolation method of [DFJP] (Section 3 below), together with an embedding result due to R. Pol and S. Gul'ko [P] (Section 4 below). Next we exploit the fact that $F^{*}$ has the RNP plus the countability assumption appearing in the very definition of $\Sigma(\Gamma)$ to construct a projectional resolution $\left(P_{\alpha}\right)_{\alpha \in I}$ of the identity in $F$ such that the adjoint maps $\left(P_{\alpha}^{*}\right)_{\alpha \in I}$ are a projectional resolution of the identity for $F^{*}$. This argument is taken from [V] and will be given in Section 4 below.

Finally, we shall see that $\left(P_{\alpha}^{*}\right)_{\alpha \in I}$ leads to a weak*-to-weak continuous injection from $F^{*}$ into $c_{0}(\Gamma)$, thus showing that ball $\left(F^{*}\right)$ (and therefore $K$ ) is Eberlein and the Banach space $F$ is weakly compactly generated.

3. Interpolation spaces for weak* compact subsets. Let $E$ be a Banach space and $D$ an absolutely convex and weak* compact subset of $E^{*}$. Denote by $|\cdot|$ the seminorm on $E$ dual to $D$, i.e.

$$
|x|=\sup \{\langle x, y\rangle: y \in D\},
$$

and by $(G,|\cdot|)$ the Banach space obtained by completing the equivalence classes modulo $|\cdot|$ of $E$; let $j$ be the canonical map $j: E \rightarrow G$. For $1 \leqslant p<\infty$ denote by $F_{p}$ the [DFJP]-interpolation space

and

$$
F_{p}=\left\{x \in G:\|x\|_{F_{p}}=\left(\sum_{n=1}^{\infty}\|x\|_{n}^{p}\right)^{1 / p}<\infty\right\}
$$

$$
F_{c_{0}}=\left\{x \in G:\|x\|_{F_{c_{0}}}=\max \left\{\|x\|_{n}\right\}<\infty \text { and } \lim \|x\|_{n}=0\right\}
$$

where $\|\cdot\|_{n}$ is the norm on $G$ whose unit ball is given by

$$
\operatorname{ball}\left(G,\|\cdot\|_{n}\right)={\overline{2^{n} j(\operatorname{ball}(E,\|\cdot\|))+2^{-n} \operatorname{ball}(G,|\cdot|)}}^{(G,|\cdot|)} \text {. }
$$

We shall need the following notion:

DEFINITION. A class $\mathscr{E}$ of compact Hausdorff spaces is called a perfect class if $\mathscr{E}$ is stable under taking continuous images, countable products, and closed subspaces.

Typical examples of perfect classes are Eberlein [L], Talagrand [T], Gul'ko $[\mathrm{T}]$, Corson compact spaces (see the survey papers of $[\mathrm{Ne}]$ and $[\mathrm{P}]$ for a detailed account of all these examples), fragmented compact spaces ([N], $[R]$ ), the class $\mathbb{C}$ considered by Stegall [S3], etc. The fact that the continuous image of an Eberlein compact is also Eberlein was proved by Benyamini, Rudin and Wage [BRW], and for Corson compact spaces the result is due to Gul'ko [Gu1], Michael and Rudin [MR]. Let us mention here that it is an open problem whether a continuous image of a Radon-Nikodym compact is also Radon-Nikodym.

Our main objective here is to prove the following:

Proposition 3.1. Let $E$ be a Banach space and $D$ an absolutely convex and weak* compact subset of $E^{*}$ that belongs to a perfect class $\mathbb{E}$. Then the unit ball of $\left(F_{p}\right)^{*}$ as well as the unit ball of $\left(F_{c_{0}}\right)^{*}$ equipped with the weak* topology belong to $\mathbb{E}$.

Proof. Define, for $1 \leqslant p<\infty$, and

$$
\Sigma_{p}=\Sigma_{p}\left(G,\|\cdot\|_{n}\right)=\left\{\ddot{x}=\left(x_{n}\right): x_{n} \in G \text { and }\|\vec{x}\|=\left(\sum_{n=1}^{\infty}\left\|x_{n}\right\|_{n}^{p}\right)^{1 / p}<\infty\right\}
$$

$$
\begin{aligned}
\Sigma_{c_{0}} & =\Sigma_{c_{0}}\left(G,\|\cdot\|_{n}\right) \\
& =\left\{\ddot{x}=\left(x_{n}\right): x_{n} \in G,\|\vec{x}\|=\sup \left\{\left\|x_{n}\right\|_{n}\right\}<\infty \text { and } \lim \left\|x_{n}\right\|=0\right\} .
\end{aligned}
$$

By definition $F_{p}$ (resp. $F_{c_{0}}$ ) is isometrically isomorphic to the diagonal of $\Sigma_{p}$ (resp. $\Sigma_{c_{0}}$ ), i.e. those $\left(x_{n}\right) \in \Sigma_{p}$ such that $x_{1}=x_{2}=\ldots$ Hence the unit ball of $\left(F_{p}\right)^{*}$ (resp. $\left.\left(F_{c_{0}}\right)^{*}\right)$ is a continuous image of the unit ball of $\left(\Sigma_{p}\right)^{*}$ (resp. $\left.\left(\Sigma_{c_{0}}\right)^{*}\right)$ with the weak* topology, and it therefore will suffice to show that the unit balls of $\left(\Sigma_{p}\right)^{*}$ and $\left(\Sigma_{c_{0}}\right)^{*}$ equipped with their weak* topologies are in the class $\&$.

Note that the identity on $G$ induces a continuous injection from $\Sigma_{1}$ into $\Sigma_{p}$ for $1 \leqslant p<\infty$ and into $\Sigma_{c_{0}}$ of norm 1 and with dense range. Hence the unit balls of $\left(\Sigma_{p}\right)^{*}$ and $\left(\Sigma_{c_{0}}\right)^{*}$ are weak* homeomorphic to subsets of the unit ball of $\left(\Sigma_{1}\right)^{*}$ and it therefore will suffice to show that the latter ball is in $\mathscr{E}$.

Finally, observe that the dual unit ball of $\left(G,\|\cdot\|_{n}\right)^{*}$ may naturally be identified with a subset of $2^{n} D$ and therefore belongs to 8 . As (ball $\left(\Sigma_{1}\right)^{*}$, weak*) is homeomorphic to $\prod_{n=1}^{\infty}\left(\right.$ ball $\left(G,\|\cdot\|_{n}\right)^{*}$, weak*) we conclude that 
(ball $\left(\Sigma_{1}\right)^{*}$, weak ${ }^{*}$ ) is in $\mathscr{E}$, thus finishing the proof.

COROLlary 3.2. Let $K$ be a weak* compact subset of a dual Banach space $E^{*}$ which is norm fragmented and such that its weak ${ }^{*}$ closed absolutely convex hull $D$ belongs to a perfect class $\mathscr{E}$ of compact spaces. Then for every $1<p<\infty$ the spaces $F_{p}$ and $F_{c_{0}}$ obtained by the above interpolation are Asplund and their dual unit balls belong to $\mathscr{E}$.

Proof. The fact that $D$ is norm fragmented if $K$ is so follows from a result of I. Namioka [N, Th. 2.5], whence a theorem of C. Stegall [S2] applies to yield that $F_{p}$ as well as $F_{c_{0}}$ is an Asplund space (compare also [N]). By Proposition 3.1 above the unit balls of $\left(F_{p}\right)^{*}$ and $\left(F_{c_{0}}\right)^{*}$ with their weak* topologies are also in 8 . $m$

4. Banach spaces with weak* Corson compact dual unit balls. A topological space $X$ belongs to the class $\Sigma$ if there is a set $\Gamma$ and a continuous injection of $X$ into $\Sigma(\Gamma)$. Banach spaces with weak* dual in $\Sigma$ have been introduced and studied by Valdivia [V]. Namely, he proves that any Banach space $E$ with $\left(E^{*}, \sigma\left(E^{*}, E\right)\right)$ in $\Sigma$ admits a projectional resolution of identity, and in the case of $E$ being an Asplund space too, the projectional resolution of identity can be chosen to be shrinking and the Banach space $E$ is weakly compactly generated. In [V], the hypothesis was used that $\left(E^{*}, \sigma\left(E^{*}, E\right)\right)$ is in $\Sigma$ while in the present context the hypothesis that (ball $\left.\left(E^{*}\right), \sigma^{*}\right)$ is in $\Sigma$ (i.e. is a Corson compact) is natural. Fortunately the two concepts coincide as will be shown in the subsequent proposition, which uses arguments of R. Pol and S. Gul'ko ([P], [Gu2]).

Proposition 4.1. The weak dual $\left(E^{*}, \sigma\left(E^{*}, E\right)\right)$ of a Banach space $E$ is in the class $\Sigma$ if, and only if, the dual ball is a weak* Corson compact space. Moreover, in that case there is a set $\left\{e_{\gamma}: \gamma \in \Gamma\right\} \subset E$ such that the mapping $T$ from $E^{*}$ into $\mathbf{R}^{T}$ defined by $T(f)=\left(\left\langle e_{\gamma}, f\right\rangle\right\rangle_{\gamma \in \Gamma}$ is injective, weak ${ }^{*}$-to-pointwise continuous, and such that $T\left(E^{*}\right) \subset \Sigma(\Gamma)$.

Proof. Let $V$ denote the unit ball of $E^{*}$ endowed with the weak* topology. Suppose that $V$ is Corson compact. A theorem of R. Pol says that $C_{\mathrm{p}}(V)$ is the continuous image of some closed subset of the product space $L(\tau)^{\mathrm{N}}$, where $L(\tau)$ is a union of a discrete space $T$ of cardinality $\tau$ and a "point at infinity" $p$ whose neighbourhoods are of the form $\{p\} \cup(T \backslash C)$, where $C$ is countable [P]. The Banach space $E$ with the weak topology is a closed subspace of $C_{\mathrm{n}}(V)$. by Grothendieck's completeness theorem. Therefore there is a closed subspace $F$ of $L(\tau)^{\mathrm{N}}$ and a continuous map $\psi$ from $F$ onto $\left(E, \sigma\left(E, E^{*}\right)\right)$. If we apply the results of Gul'ko and Pol at this point [P, Prop. 1.4, p. 23], we find a set $\Gamma$ and a continuous linear injection $S$ from $C_{\mathrm{p}}(F)$ into $\Sigma(\Gamma)$. If we denote by $R$ the map from $C_{\mathrm{p}}\left(E, \sigma\left(E, E^{*}\right)\right)$ into $C_{\mathrm{p}}(F)$ defined by $R(f)=f \circ \psi$, the composition $S \circ R$ is injective and continuous from $C_{\mathrm{p}}\left(E, \sigma\left(E, E^{*}\right)\right)$ into $\Sigma(\Gamma)$. The restriction to the dual $E^{*}$ gives us a linear, continuous and one-to-one map from
$\left(E^{*}, \sigma\left(E^{*}, E\right)\right)$ into $\Sigma(\Gamma)$. The linearity of this map means that it is defined through a set of coordinate functionals $\left\{e_{y}: \gamma \in \Gamma\right\} \subset E$. .

For the convenience of the reader we shall include here a proof of the fact that an Asplund space with weak* Corson compact unit dual ball is weakly compactly generated, which is a corollary of the last proposition and the results of [V]. Our proof follows the arguments in [V] (compare also [OV]). Let us also remark here that our Theorem $B$ is more general than this particular result. Indeed, if we apply Theorem 4.2 to Banach spaces of continuous functions on a compact set $K$ we only find that every scattered and Corson compact space is an Eberlein compact ([A], [V]).

THEOREM 4.2. If an Asplund space $E$ has a Corson compact weak* unit dual ball, then $E$ is weakly compactly generated.

Proof. Let $T$ be the mapping determined by $\left\{e_{\gamma}: \gamma \in \Gamma\right\}$, defined in the last proposition. We shall consider the multivalued map

$$
\varphi: E^{*} \rightarrow 2^{E}, \quad \varphi(f)=\left\{e_{\gamma}:\left\langle e_{\gamma}, f\right\rangle \neq 0\right\},
$$

which has the following properties:

( $\varphi 1)$ For every $f$ in $E^{*}$ the set $\varphi(f)$ is countable.

In the duality $\left(E, E^{*}\right)$, for a subset $C$ of $E$ (resp. of $E^{*}$ ) we shall denote by $C^{\perp}$ the orthogonal of $C$ in $E^{*}$ (resp. in $E$ ). With this notation:

$(\varphi 2) \quad$ For every subset $B \subset E^{*}, \varphi(B)^{\perp} \cap \bar{B}^{\text {weak* }}=\{0\}$ :

Indeed, if $f$ is in the weak ${ }^{*}$ closure of $B$ and $f \neq 0$, there is some $\gamma$ in $\Gamma$ such that $\left\langle e_{y}, f\right\rangle \neq 0$, hence $\left\langle e_{y}, b\right\rangle \neq 0$ for some $b$ in $B$ and $e_{y} \in \varphi(B)$, so $f$ does not belong to $\varphi(B)^{\perp}$.

We shall say that a pair of Q-linear subspaces $A$ in $E$ and $B$ in $E^{*}$ is a norming pair when for every $x$ in $A$ and every $f$ in $B$ we have

$$
\begin{aligned}
& \|x\|=\sup \left\{|\langle x, g\rangle|: g \in \operatorname{ball}\left(E^{*}\right) \cap B\right\}, \\
& \|f\|=\sup \{|\langle y, f\rangle|: y \in \operatorname{ball}(E) \cap A\} .
\end{aligned}
$$

Whenever we have a norming pair $(A, B)$ in a Banach space $E$, the norm closures $\bar{A}$ and $\bar{B}$ are a norming pair too. Observe that if $(A, B)$ is a norming pair, then for every $x \in A$ and $y \in B^{\perp}$ we have the inequality $\|x\| \leqslant\|x+y\|$, analogously for every $f \in A^{\mathcal{L}}$ and $g \in B,\|g\| \leqslant\|g+f\|$.

$(\varphi 3)$ For every norming pair $(A, B)$ in $E$ with $\varphi(B) \subset A$ there is a norm one projection $P$ in $E$ with range $A$ and kernel $B^{\perp}$.

Indeed, the hypothesis we have together with $(\varphi 2)$ implies that the weak* closure of $B$ meets $A^{\perp}$ only in zero. So $A+B^{\perp}$ is dense in the Banach space $E$. The above inequalities give us the projection $P$. 
Following Fabian and Godefroy [FG] we shall now consider the multivalued map $J$ from $E$ into ball $\left(E^{*}\right)$ defined by

$$
J(x)=\left\{f \in \operatorname{ball}\left(E^{*}\right):\langle x, f\rangle=\|x\|\right\} .
$$

Since $E^{*}$ has RNP, the selection theorem of Jayne and Rogers, [JR, Th. 8] gives a sequence $\left(\psi_{n}\right)$ of norm-to-norm continuous functions from $E$ into $E^{*}$ which pointwise converges in the norm topology to a mapping $\psi_{0}$ that satisfies $\left\langle x, \psi_{0}(x)\right\rangle=\|x\|$ for every $x \in E$. We now define the multivalued map

$$
\psi: E \rightarrow 2^{E^{*}}, \quad \psi(x)=\left\{\psi_{0}(x), \psi_{1}(x), \ldots\right\},
$$

which has the following properties:

$(\psi 1) \quad F o r$ every $x$ in $E$ the set $\psi(x)$ is countable.

$(\psi 2) \quad F$ or every closed subspace $V$ of $E$ the norm closed linear span of the set $\left\{\left.f\right|_{V}: f \in \psi(V)\right\}$ coincides with $V^{*}$.

This property has been used in [FG] in order to obtain a projectional resolution of the identity in the dual of any Asplund space. The proof relies on the weak* version of James' compactness theorem [FG, main theorem].

$(\psi 3)$ For every norming pair $(A, B)$ in $E$ with $\psi(A) \subset B$ there is a norm one projection $Q$ in $E^{*}$ with range $\bar{B}^{\|} \|$and kernel $A^{\perp}$.

This follows from $(\psi 2)$ since the restriction map from $E^{*}$ onto $\left(\bar{A}^{\|\|}\right)^{*}$ maps $B$ onto $\left(\bar{A}^{\|} \|^{*}\right.$.

We have now assembled all the ingredients to construct a shrinking resolution of the identity in the Banach space $E$ :

Step 1. Let $\lambda$ be a cardinal number and let $A_{0} \subset E$ and $B_{0} \subset E^{*}$ be two infinite subsets with $\left|A_{0}\right| \leqslant \lambda$ and $\left|B_{0}\right| \leqslant \lambda$. There is a norming pair $(A, B)$ in E with

$$
A_{0} \subset A \subset E, \quad B_{0} \subset B \subset E^{*}, \quad|A| \leqslant \lambda, \quad|B| \leqslant \lambda,
$$$$
\varphi(B) \subset A, \quad \psi(A) \subset B .
$$

We proceed by induction: Let $p \in \mathbf{N}$ and suppose that for every integer $m$ with $0 \leqslant m \leqslant p$, we have subsets

$$
A_{m} \subset E, \quad B_{m} \subset E^{*}, \quad\left|A_{m}\right| \leqslant \lambda, \quad\left|B_{m}\right| \leqslant \lambda .
$$

We write $C_{m}$ and $D_{m}$ for the Q-linear spans of $A_{m}$ and $B_{m}$, respectively. Define

$$
B_{p+1}:=D_{p} \cup\left\{\psi(x): x \in C_{p}\right\}, \quad A_{p+1}:=C_{p} \cup\left\{\varphi(f): f \in D_{p}\right\} \cup N_{p},
$$

where $N_{p}$ is a subset of cardinality less than or equal to $\lambda$ which norms every element of $D_{p}$. If we take $A=\bigcup\left\{A_{n}: n=0,1, \ldots\right\}$ and $B=\bigcup\left\{B_{n}: n=\right.$ $0,1, \ldots\}$, then $(A, B)$ is a norming pair with the required properties.

Step 2. For a norming pair $(A, B)$ as above there is a norm one projection
$P$ in $E$ with range equal to the norm closure of $A$ and with kernel $B^{\perp}$. Moreover, the adjoint projection $P^{*}$ has range equal to the norm closure of $B$ in $E^{*}$.

That follows from properties $(\varphi 3)$ and $(\psi 3)$ above.

We shall say that the projection $P$ is based on the norming pair $(A, B)$ to summarize the above situation.

Step 3. If $\left\{x_{\alpha}: \alpha<\mu\right\}$ is a dense subset of $E$, where $\mu$ is the first ordinal number with $|\mu|=\operatorname{dens}(E)$, then there is a projectional resolution of identity $\left\{P_{\alpha}: \omega_{0} \leqslant \alpha \leqslant \mu\right\}$ in $E$ where every $P_{\alpha}$ is based on a norming pair $\left(A_{\alpha}, B_{\alpha}\right)$ such that

$$
\begin{gathered}
\left|A_{\alpha}\right| \leqslant|\alpha|, \quad\left|B_{\alpha}\right| \leqslant|\alpha|, \quad\left\{x_{\beta}: \beta<\alpha\right\} \subset A_{\alpha} \quad \text { for every } \alpha \in\left[\omega_{0}, \mu\right], \\
A_{\beta} \subset A_{\alpha} \text { and } B_{\beta} \subset B_{\alpha} \quad \text { if } \omega_{0} \leqslant \beta \leqslant \alpha \leqslant \mu, \\
A_{\alpha}=\bigcup\left\{A_{\beta}: \omega_{0} \leqslant \beta<\alpha\right\} \text { and } B_{\alpha}=\bigcup\left\{B_{\beta}: \omega_{0} \leqslant \beta<\alpha\right\}
\end{gathered}
$$

for every limit ordinal $\alpha>\omega_{0}$

and the adjoint projections $\left\{P_{\alpha}^{*}: \omega_{0} \leqslant \alpha \leqslant \mu\right\}$ are a projectional resolution of the identity in the dual space $E^{*}$.

Let $A_{0}$ be equal to $\left\{x_{\alpha}: \alpha<\omega_{0}\right\}$ and $B_{0}$ be equal to $\psi\left(A_{0}\right)$. Step 1 gives us a norming pair $\left(A_{\omega_{0}}, B_{\omega_{0}}\right)$ of countable $\mathbf{Q}$-linear spaces and step 2 a norm one projection $P_{\omega_{0}}$ from $E$ onto the norm closure of $A_{\omega_{0}}$ with adjoint $P_{\omega_{0}}^{*}$ from $E^{*}$ onto the norm closure of $B_{\omega_{0}}$. We shall proceed by transfinite induction to construct the other projections. Take $\omega_{0}<\alpha<\mu$ and assume that for $\omega_{0} \leqslant \beta<\alpha$ we have defined the projection $P_{\beta}$ based on the norming pair $\left(A_{\beta}, B_{\beta}\right)$, with the corresponding properties. If $\alpha=\gamma+1$, we take $A_{0}$ equal to $A_{\gamma} \cup\left\{x_{\gamma}\right\}$ and $B_{0}$ equal to $\psi\left(A_{\gamma}\right) \cup B_{\gamma}$. Another application of step 1.produces a norming pair $\left(A_{\alpha}, B_{\alpha}\right)$ of $\mathbf{Q}$-linear subspaces of cardinality less than or equal to $|\alpha|$, and step 2 gives us a norm one projection $P$ from $E$ onto the norm closure of $A_{\alpha}$ with adjoint $P_{\alpha}^{*}$ from $E^{*}$ onto the norm closure of $B_{\alpha}$. If $\alpha$ is a limit ordinal, we take

$$
A_{\alpha}=\bigcup\left\{A_{\beta}: \omega_{0} \leqslant \beta<\alpha\right\}, \quad B_{\alpha}=\bigcup\left\{B_{\beta}: \omega_{0} \leqslant \beta<\alpha\right\} .
$$

$\left(A_{\alpha}, B_{\alpha}\right)$ is a norming pair of $\mathbf{Q}$-linear subspaces of cardinality at most $|\alpha|$ with $\varphi\left(B_{\alpha}\right) \subset A_{\alpha}$ and $\psi\left(A_{\alpha}\right) \subset B_{\alpha}$. Therefore, step 2 produces a norm one projection $P_{\alpha}$ from $E$ onto the norm closure of $A_{\alpha}$ with adjoint $P_{\alpha}^{*}$ from $E^{*}$ onto the norm closure of $B_{\alpha}$. Finally, $P_{\mu}$ is the identity operator and we have finished the construction.

This shrinking resolution implies that $E$ is weakly compactly generated as we are going to show in the next steps that follow the well known argument of [AL].

Step 4. There is a set $I$ and a continuous linear injection $T$ from $E^{*}$ into $c_{0}(I)$ which is one-to-one and weak*-to-weak continuous. 
Again we shall proceed by transfinite induction on the density character of the Banach space $E_{\text {i }}$ If $E$ is separable there is nothing to prove. Suppose the result is true for every space with density character strictly less than the density character of $E$. Let $\left\{P_{\alpha}: \omega_{0} \leqslant \alpha \leqslant \mu\right\}$ be the shrinking resolution of identity constructed above. For every $\alpha, P_{\alpha}(E)$ is an Asplund space with weak* Corson compact unit dual ball and $\operatorname{dens}\left(P_{\alpha}(E)\right) \leqslant|\alpha|<\operatorname{dens}(E)=|\mu|$. By the induction hypothesis there is a weak*-to-weak linear injection $T_{\alpha}$ from $P_{\alpha}^{*}\left(E^{*}\right)$ into $c_{0}\left(I_{\alpha}\right)$ for some set $I_{\alpha}$ with $\left\|T_{\alpha}\right\| \leqslant 1$. If $I$ denotes the disjoint union of the family of sets $\left\{I_{\omega_{0}}, I_{\alpha+1}: \omega_{0} \leqslant \alpha<\mu\right\}$, we define the operator $T$ from $E^{*}$ into $I^{\infty}(I)$ by

$$
\begin{array}{ll}
(T f)(\gamma):=T_{\omega_{0}}\left(P_{\omega_{0}}^{*}(f)\right)(\gamma) & \text { if } \gamma \in I_{\omega_{0}}, \\
(T f)(\gamma):=\frac{1}{2} T_{\alpha+1}\left(\left(P_{\alpha+1}^{*}-P_{\alpha}^{*}\right)(f)\right)(\gamma) & \text { if } \gamma \in I_{\alpha+1} .
\end{array}
$$

The fact that $T\left(E^{*}\right) \subset c_{0}(I)$ follows from the fact that $\left\{P_{\alpha}^{*}: \omega_{0} \leqslant \alpha \leqslant \mu\right\}$ is a resolution of the identity in $E^{*}$. Indeed, given $\varepsilon>0$ and $f$ in $E^{*}$ the set $\left\{\alpha: \omega_{0} \leqslant \alpha<\mu\right.$ and $\left.\left\|\left(P_{\alpha+1}^{*}-P_{\alpha}^{*}\right)(f)\right\|>\varepsilon\right\}$ is finite because for every limit ordinal $\beta$ we have \|\| $\lim \left\{P_{\alpha}^{*}(f): \alpha<\beta\right\}=P_{\beta}^{*}(f)$

Step 5. (ball $\left(E^{*}\right)$, weak*) is affinely homeomorphic to a weakly compact subset of $c_{0}(I)$ and $E$ is weakly compactly generated.

Indeed, the above mapping $T$ is weak*-to-weak continuous. The conclusion now follows from a result of Lindenstrauss [L, Th. 3.3].

5. Radon-Nikodym and Corson compact spaces. Now, we are ready to prove Theorem $A$ stated in the introduction:

Proof of Theorem A. Suppose $K$ is Radon-Nikodym and Corson compact. By definition $K$ is homeomorphic to a weak* compact subset of the dual $E^{*}$ of a Banach space such that the dual norm fragments $K$. It is known that every regular Borel probability measure on $K$ has separable support (see for instance [S], or [JNR]), and that for a Corson compact space $K$ with this property the unit ball of $C(K)^{*}$ is a Corson compact space [AMN; Th 3.5]. Therefore the weak* closed absolutely convex hull $D$ of $K$ is a Corson compact, since it is the continuous image of the weak* unit ball of $C(K)^{*}$. We can now apply Corollary 3.2 to the perfect class of Corson compact spaces and find an Asplund space $F$ with weak* Corson compact dual unit ball such that $K$ is homeomorphic to a weak ${ }^{*}$ compact subset of the dual $F^{*}$. Theorem 4.2 says that $F$ is weakly compactly generated and thus $K$ is Eberlein compact.

Remark. Let us mention that the above argument can be simplified if we replace the assumption " $K$ is Corson" by the stronger assumption " $K$ is Gul'ko". Indeed, as it is known that for a Gul'ko compact space $K$, the weak" unit ball of $C(K)^{*}$ is Gul'ko compact we do not have to refer to the results of [AMN] in this case. The interpolation space $F$ is then weakly countably determined (apply Corollary 3.2 to the perfect class of Gul'ko compact spaces and note that $F$ is weakly countably determined iff (ball $\left.\left(F^{*}\right), \sigma^{*}\right)$ is Gul'ko [T]). Hence we do not need the full strength of 4.2 above and can instead apply a result of M. Fabian [F] to conclude as above that $F$-is weakly compactly generated.

We now turn to the proof of Theorem $B$ stated in the introduction.

Proof of Theorem B. Suppose that $E$ is a Banach space such that (ball $\left.\left(E^{*}\right), \sigma^{*}\right)$ is Corson compact and such that there is a continuous linear map from an Asplund space $X$ into $E$ with dense range. By Theorem A, (ball $\left.\left(E^{*}\right), \sigma^{*}\right)$ is Eberlein compact. Note; however, that this only would allow us to conclude that $E$ is a subspace of a weakly compactly generated Banach space. But an inspection of the proof of Theorem A shows that (ball $\left.\left(E^{*}\right), \sigma^{*}\right)$ is in fact affinely homeomorphic to a weakly compact subset of $c_{0}(\Gamma)$. Hence by a theorem of Lindenstrauss [L, Th, 3.3] we may conclude that $E$ is weakly compactly generated, thus completing the proof of Theorem B.

Remark. It seems to have been unknown so far whether weakly $K$-analytic or - more generally - weakly countably determined Banach spaces necessarily " are GSG. Of course, Theorem B above shows that this is not the case: For any Corson compact space $K$ which fails to be Eberlein compact (e.g. Talagrand's space) the space $C(K)$ fails to be GSG.

\section{References}

[A] K. Alster, Some remarks on Eberlein compacts, Fund. Math. 104 (1979), 43-46.

[AL] D. A mir and J. Lindenstrauss, The structure of weakly compact sets in Banach spaces, Ann. of Math. 88 (1968), 35-46.

[AMN] S. Argyros, S. Mercourakis and S. Negrepontis, Functional-analytic properties of Corson-compact spaces, Studia Math. 89 (1988), 197-229.

[BRW] Y. Benyamin M. E Rudin and M. Wage, Continuous images of weakly compact subsets of Banach spaces, Pacific J. Math. 70 (1977), 309-324.

[DFJP] W. J. Davis, T. Figiel, W. B. Johnson and A. Pelczyński, Factoring weakly compact operators, J. Funct. Anal. 17 (1974), 311-327.

[F] M. Fabian, Each weakly countably determined Asplund space admits a Fréchet differentiable norm, Bull. Austral. Math. Soc. 36 (1987), 367-374.

[FG] M. Fabian and G. Godefroy. The dual of every Asplund space admits a projectional resolution of the identity, Studia Math. 91 (1988), 141-151.

[G] Goc. 100 (1987) G. Grue

[Gu1.] S. P. Gul'ko, On properties of subsets of E-products, Dokl. Akad. Nauk SSSR. 237 (1977), 505. 508 (in Russian).

[Gu2] - On properties of some function spaces, ibid. 243 (1978), $839-842$ (in Russian).

[Gu3] - The structure of spaces of continuous functions and their hereditary paracompactness, Russian Math. Surveys 34 (1979), 36-44

[GM] G Goussoub and B. Maurey, $H_{3}$-embeddings in Hilbert space and optimization on

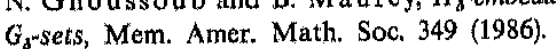

[JR] J. E. Jayne and C. A. Rogers, Borel selectors for upper semi-continuous set-valued maps, Acta Math. 155 (1985), 41-79. 


\section{INFORMATION FOR AUTHORS}

[JNR] J. E. Jayne, I. Namioka and C. A. Rogers, Norm fragmented weak-* compact sets, in: Seminar on Functional Analysis 2, University of Murcia, to appear.

[K] P. S. Kenderov, A Talagrand compact space which is not a Radon-Nikodym space (An example of E. A. Reznichenko), manuscript.

[L] J. Lindenstra uss, Weakly compact sets, their topological properties and the Banach spaces they generate, Ann. Math. Stud. 69, Princeton Univ. Press, 1972, 235-276.

[MR] E. Michael and M. E. Rudin, $A$ note on Eberlein compacta, Pacific J. Math, 72 (1977), $487-495$.

[N] I. Namioka, Radon-Nikodym compact spaces and fragmentability, Mathematika 34 (1987), 258-281.

[Ne] S. Negrepontis, Banach spaces and topology, Ch. 23 of: Kandbook of Set-Theoretic Topology, K. Kunen and J. E. Vaughan (eds.), Elsevier, 1984, 1045-1142.

[OV] J. Orih uela and M. Valdivia, Projective generators and resolutions of identity in Banach spaces, Rev. Mat. Univ. Compl. Madrid 2 (1989), 179-199.

[P] R. Pol, on pointwise and weak topology in function spaces, preprint 4/84, Warsaw University, 1984.

[R] N. K. Ribarska, Internal characterization of fragmentable spaces, Mathematika 34 (1987), 243-257.

[S]. L. Schwartz, La propriété de Radon-Nikodym, Exp. IV-VI, Séminaire Maurey-Schwartz 1974-75, Publ. de l'Ecole Polytechnique, Paris.

[S1] C. Stegall, The Radon Nikodym property in conjugate Banach spaces, Trans. Amer. Math. Soc. 206 (1975), 213-223.

[S2] -, The Radon-Nikodym property in conjugate Banach spaces. II, ibid. 264 (1981), 507-519.

[S3] -, Applications of descriptive topology in Functional Analysis, Linz University, 1985-87.

[T] M. Talagrand, Espaces de Banach faiblement $\mathscr{K}$-analytiques, Ann. of Math. 110 (1979), 407-438.

[To] S. Todorčević, Trees and linearly ordered sets, Ch, 6 of: Handbook of Set-Theoretic Topology, K. Kunen and J. E. Vaughan (eds.), Elsevier, 1984, 235-293.

[V] M. Valdivia, Resolutions of the identity in certain Banach spaces, Collect. Mat. 39 (1988) $127-140$.

DEPARTAMENTO DE MATEMÁTICAS UNIVERSIDAD DE MURCIA Santo Cristo 1, E-30001 Murcia, Spain

INSTITUT FUR MATHEMATIK DEPARTAMENTO DE ANALISIS MATEMATICO UNIVERSIDAD DE VALENCIA Doctor Molinar 50, E-46100 Burjasot, Valencia, Spain
Manuscripts should be typed on one side only, with double or triple spacing and wide margins, and submitted in duplicate, including the original typewritten copy. Poor quality copies will not be accepted.

An abstract of not more than 200 words is required. The AMS Mathematics Subject Classification is desirable.

Formulas should be typewritten, and with the number (if any) placed in parentheses at the left margin.

A complete list of all handwritten symbols with indications for the printer should be enclosed. Special typefaces should be indicated according to the following code; script letters-by encircling the typed Roman letter in black, German letters - by typing the Roman equivalent and underlining in green, boldface letters - by straight black underlining.

No underlining is necessary for the titles, the text of theorems, the words "Theorem", etc. These will be set automatically according to the style of the journal. No titles should be written in capital letters.

Figures should be drawn accurately on separate sheets, preferably twice the size in which they are required to appear. The author should indicate in the margin of the manuscript where figures are to be inserted.

References should be arranged in alphabetical order, typed with double spacing, and styled and punctuated according to the examples given below. Abbreviations of journal names should follow Mathematical Reviews. Titles of papers in Russian should be translated into English.

Examples:

[6] D. Beck, Introduction to Dynamical Systems, Vol. 2, Progr. Math. 4, Birkhäuser, Basel 1978. [with no underlining in the manuscript]

[7] R. Hill and J. James, An index formula, J. Differential Equations 15 (1982), 197-211.

[8] J. Kowalski, Some remarks on $J(X)$, in: Algebra and Analysis, Proc. Conf. Edmonton 1973 , E. Brook (ed.), Lecture Notes in Math. 867, Springer, Berlin 1974, 115-124.

[Nov] A. S. Novikov, An existence theorem for planar graphs, preprint, Moscow University 1980 (in Russian).

Authors' affiliation should be given at the end of the manuscript.

Authors receive only page proofs (one copy). If the proofs are not returned promptly, the article will be proofread against the manuscript by the publisher and printed without the author's corrections. Authors receive 50 reprints of their articles. 